\title{
CURRENT STATUS AND FUTURE RESEARCH DIRECTIONS IN MONITORING VIGILANCE OF INDIVIDUAL OR MASS AUDIENCE IN MONOTONOUS WORKING ENVIRONMENT
}

\author{
B. F. Momin ${ }^{1}$ and Parag P. Abhyankar ${ }^{2}$ \\ ${ }^{1}$ Department of Computer Engg., WCE, Sangli, India \\ bfmomineyahoo.com \\ ${ }^{2}$ Department of Information Technology, Finolex Academy, Ratnagiri, India \\ paragabhyankar15@gmail.com
}

\begin{abstract}
Working in monotonous environment often causes lack of concentration or fatigue in an operator and many times such non-vigilance leads to accidents. Therefore early detection of fatigued state has become essential in monotonous working environments like driving vehicle, operating machines etc. Such fatigued state often gets developed gradually and can be identified by certain symptoms. Different types of symptoms help in modelling non-vigilance in different ways. This paper reviews and compares current status of research in modelling fatigue where fatigue is modelled using probabilistic models, machine learning models, finite state machine etc. The paper also presents possible future research directions in the same field like identifying non-fatigue non-vigilance mental states, extending non-vigilance monitoring for mass audience etc.
\end{abstract}

\section{KEYWORDS}

Vigilance Monitoring, Fatigue Modelling, Physiological, Behavioural and Visual Measure, Machine Learning.

\section{INTRODUCTION}

The increasing number of traffic accidents due to driver's diminished vigilance level has become a serious problem for society. The statistics show that $10 \%$ to $20 \%$ of all traffic accidents in Europe are due to driver's diminished vigilance level caused by fatigue. In the trucking industry, about $60 \%$ of fatal truck accidents are related to driver fatigue. It is the main cause of heavy truck crashes [1]. Monitoring the driver's level of vigilance and alerting the driver when he is not paying adequate attention to the road has become essential in order to prevent accidents. [1]. Detection of driver's visual attention is very important for developing automatic systems that monitor the driver's inattention, driver fatigue, and lack of sleep. A great number of fatalities occurring in motor vehicles could be avoided if these behaviours were detected.

Apart from fatigue, non-vigilance can also be caused by certain mental states. Anger, frustration, excitation or being uncomfortable with the environment is some of the mental states which can lead to decreased vigilance.

DOI: $10.5121 /$ ijsc.2012.3204 
Many times listening lectures or attending meetings becomes monotonous and the symptoms of doziness can be clearly seen in audience. There cannot be any better effectiveness measure of the talk rather than the overall vigilance of the audience. Time patterns in audience vigilance would be useful in better organization of talk contents.

This paper presents current status of research in vigilance monitoring and some possible future research direction in the same field. The paper is organized as follows. Current status of research is reviewed in Section 2; applications of vigilance monitoring are discussed in section 3. Different fatigue models are compared in section 4 . Section 5 presents possible future research direction in vigilance monitoring and paper is concluded in section 6 .

\section{LITERATURE SURVEY}

The most common reason of non-vigilance is fatigue and most of the researches in modelling non-vigilance concentrate on characterization of fatigue. Fatigued state of a person can be sensed through different measures which are mainly classified into physiological, behavioural, visual, and subjective performance measures. [2]

\subsection{Physiological Measures:}

This method has been thought to be accurate, valid, and objective to determine fatigue and sleep. Significant efforts have been made in laboratory to measure it. The popular physiological measures include the electroencephalograph (EEG). EEG is found to be useful in determining the presence of ongoing brain activity, and its measures have been used as the reference point for calibrating other measures of sleep and fatigue [2].

Electroencephalography (EEG) is the recording of electrical activity along the scalp. EEG measures voltage fluctuations resulting from ionic current flows within the neurons of the brain through multiple electrodes placed on the scalp. The frequency of brainwave in EEG is about 1$30 \mathrm{~Hz}$ and generally it can be divided into four types in terms of frequency bands, which are delta $(0-4 \mathrm{~Hz})$, theta $(4-8 \mathrm{~Hz})$, alpha $(8-13 \mathrm{~Hz})$, and beta $(13-20 \mathrm{~Hz})$. The alpha rhythm is present during a relaxed state and beta activity has been reported to be present during an alert state. Delta waves have been shown to be present during transition to drowsiness and during sleep. Theta rhythm is associated with a variety of psychological states including hypnologic imagery, and low levels of alertness during drowsiness and sleep and as such has been associated with decreased information processing. So alpha, beta and delta rhythm are independent and strongly related to fatigued state, so they three are selected as studying indicators [21].

Electroencephalogram (EEG) [10-20][25] is the most commonly studied signal for vigilance estimation. In most of the EEG-based vigilance research, EEG data of certain people is captured for experimentation and is labelled as per the vigilance states by some observer. Then different machine learning techniques can be applied on such data to find co-relation between EEG signal and observed fatigue state.

The work in [12] claims that most existing methods have focused on employing supervised learning methods to estimate vigilance states. However, till now, there is no standard criterion for vigilance scale labelling, and the existing vigilance labelling methods are complex, expensive and sometimes unreliable and therefore choose clustering methods to mine the latent distribution of EEG for vigilance estimation. The work extracts features in EEG using spatial filter and further clusters it into 4 clusters as wakefulness (W), middle state 1 (M1), middle state 2 (M2), and sleepiness (S). 
The paper [13] proposes a system that combines electroencephalogram (EEG) power spectra estimation, independent component analysis and fuzzy neural network models to estimate subjects' cognitive state in a dynamic virtual-reality-based driving environment. It first uses the independent component analysis (ICA) to remove artifacts and extract possible sources of brain activities from continuous EEG recordings. After calculating power spectrum of each ICA components, it correlates the information between the human alertness and the ICA power spectra. Here human alertness is given by a performance index (driving error expressed as deviation between the center of the vehicle and the center of the lane). They select the alpha-band power in two major ICA components with the maximum correlation coefficients for individual subject as the input features of the estimation models. The correlation between subject's driving performance index (SDPI) and 33-ICA components are calculated to select the most useful representative components. The highest correlated 2-ICA-components power spectra were selected. Then the selected alpha-band features are fed to the self-constructing neural fuzzy inference network (SONFIN) to learn the relationship between the EEG spectra and driving performance.

This paper [14] investigates the use of RF (Random Forest) to select the key EEG features from a broad pool of available features, for the purpose of multilevel mental fatigue EEG classification. The EEG data were labelled to 5-level mental fatigue. EEG data passed through the feature extraction windows, with one window for each EEG channel. The length of each feature extraction window was $2 \mathrm{~s}$ (or 334 EEG samples), with half second (or 84 EEG samples) time interval between two adjacent calculations of feature extraction. Four features were chosen to characterize the power spectral density. As a result, in total 304 quantitative EEG features (4 kinds of features, 19 channels, and 4 frequency bands) were extracted. RF is used as Machine Learning Technique for classification / recognition of features.

The work in [11], senses different physiological parameters like galvanic skin resistance (GSR), heart rate variability (HRV), body temperature (THE) and in the context vehicle driving, different mechanical data like steering wheel positioning, brake pedal positioning etc of driver by sensors put on steering wheel. A fuzzy logic classifier trained with offline statistical results is used to classify the vigilance state of driver. The classifier continuously monitors the data to detect a possible decrease in driver vigilance. If the driver is found to be sleepy, then the system is put on alert to focus on detecting a possible sleep attack and to alert the driver.

\subsection{Behavioural Measures:}

Behavioural Measures [2][25][23] are also accurate and objective. This category of devices, most commonly known as acti-graph, is used to measure sleep based on the frequency of body movement. The number of body movement recorded during a specified time period, or epoch, has been found to significantly correlate with the presence of sleep and has a significant correlation with EEG.

In [23], jerk profiles for the machine-human interfaces of vehicle are sensed as measures for assessing vigilance of the vehicle driver. Responding to the stimulus was considered as sign of vigilance. The work in [26] claims that short pauses in performances are more indicative measures of vigilance.

\subsection{Visual Measures:}

An increasing research interest has focused on developing systems that detect the visual facial feature changes associated with fatigue with a video camera. These facial features include eyes, 
head position, face, or mouth. This approach is non-intrusive and becomes more and more practical with the rapid development of camera and computer vision technology [1-9][24].

People in fatigue exhibit certain visual behaviours that are easily observable from changes in facial features like the eyes, head, and face. Visual behaviours that typically reflect a person's level of fatigue include eyelid movement, head movement, gaze, and facial expression. Various studies have shown that eyelid activities are strongly related with level of vigilance, intention, and needs. Percentage of eyelid closure (PERCLOS) has been found to be the most reliable and valid measure of a person's alertness level among many drowsiness detection measures. PERCLOS measures the percentage of eyelid closure over the pupil over time and reflects slow eyelid closures (droops). Another potentially good fatigue indicator is the average eye closure and opening speed (AECS). Since eye opening/closing is controlled by the muscle near the eyes, a person in fatigue may open/close eyes slowly due to either tired muscles or slower cognitive processing. Other potentially good fatigue parameters include various parameters that characterize pupil movement, which relates to one's gaze and his/her awareness of the happenings in surroundings. The movement of a person's pupil (gaze) may have the potential to indicate one's intention and mental condition. For example, for a driver, the nominal gaze is frontal. Looking at other directions for an extended period of time may indicate fatigue or inattention. Furthermore, when people are drowsy, their visual awareness cannot cover a wide enough area, concentrating on one direction. Hence, gaze (deliberate fixation) and saccade eye movement may contain information about one's level of alertness. Besides eye activities, head movement like nodding or inclination is a good indicator of a person's fatigue or the onset of a fatigue. It could also indicate one's attention. Head movement parameters such as head orientation, movement speed, frequency, etc. could potentially indicate one's level of vigilance. Finally, facial expression may also provide information about one's vigilance. For example, a typical facial expression that indicates the onset of fatigue is yawning. The major benefits of the visual measures are that they can be acquired non-intrusively [2].

Most of the fatigue detection work finds eyes as an interesting and useful visual measure, and fatigue is detected by detecting geometries of eyes, iris. The eyes detection method in [9] uses new circle detection operator and then uses neural network to validate the detected regions as eyes. An appearance based model in [3] uses Support Vector Machine to detect eyes and needs large number of training sets composed of different objects, orientations, light conditions, and eye closure degree. The Hypo-Vigilance Detection based on Eyelid Behaviour [6] detects first face in the image and then percentage of eye closure (PERCLOS) and eyelid distance changes (ELDC) are computed for fatigue detection and eye closure rate (CLOSNO) is used for distraction detection. Finally, the computed values are compared with some simple threshold to estimate driver hypo-vigilance.

Work in [5] is based on fact the "Variations in the pupillary size which was known controlled by the autonomic nervous system were found to be positively correlated with the level of vigilance in healthy normal subjects'. It computes the size of pupil by finding its minimum closing rectangle and finds variation in pupil size over time to detect fatigue.

Work in [1] detects eyes and tracts pupils. It also associates state with every eye status as CLOSED, CLOSING etc and changes state based on certain observation in eyes. For example when the pupil ratio is above $80 \%$ of its nominal size or the pupils are lost, being in the CLOSING_STATE, an FSM transition to the CLOSED_STATE is provoked, which means that the eyes are closed. A new detection of the pupils from the CLOSED_STATE produces a change to the OPENING_STATE. 
In [2] probabilistic model (Bayesian model) of fatigue is presented. Fatigue is modelled as the target hypothesis variable that we intend to infer while other contextual factors, which could cause fatigue (e.g. sleep quality, circadian, work condition, work environment, and physical condition), and visual cues, which are symptoms of fatigue, are information variables. On the other hand, when a person is fatigued, he/she tends to exhibit various visual behaviours that deviate from the nominal behaviours. The behaviours that typically capture the cognitive state of a person include eye movement, head movement, and facial expression. The Conditional Probability Tables (CCPTs) required for Bayesian Mode is constructed using several series of large-scale subjective surveys.

If $H$ is hypothesis variable, $o$ is observed evidence and $s$ are hidden variables, $\mathrm{H}$ can be inferred with following probability.

$$
P\left[H \sqcap \propto \sum_{s} P[H, o, s \sqsubset\right.
$$

Since fatigue is developed over period of time, in [2], it is actually modelled as Dynamic Bayesian Network (DBN). If $T$ is a time boundary, hidden variables $S=\left\{s_{0}, \ldots, s_{T-1}\right\}$, observations $O=\left\{o_{0}, \ldots, o_{T-1}\right\}$ and hypothesis $H=\left\{h_{0}, \ldots, h_{T-1}\right\}$, the probability distribution of DBN expressed as following

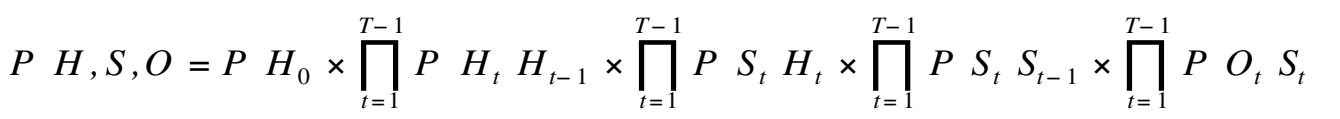

as $H$ and $S$ at each point in time depends also on $H$ ans $S$ at previous point in time respectively.

Different eye features are proposed in [6] like blink duration, eye closure, blinking rate, head nodding frequency etc. Finally drowsiness classification is defined as to combine these different features to a single continuous-valued drowsiness measure or the discrete classes awake, questionable and drowsy. An artificial neural network (ANN) was used for classification.

Yawning is used as fatigue measure in [7] and it detect yawing by detecting first mouth in an image and then its size and shape as increasing or decreasing.

In [8], the face and features of the face like eyes, mouth, and facing direction are identified and tracked which can be used for driver's fatigue detection.

\section{APPLiCATIONS OF FATIGUE DETECTION}

Generally, applications of all of the efforts on the fatigue detection can be classified in following groups [2].

\subsection{Sensing readiness / fitness-for-duty:}

This type of effort is to assess the vigilance capacity of an operator before the work is performed. The main is to sense whether the operator is fit for the duration of the duty period, or at the start of an extra period of work [2]. 


\subsection{Measuring vehicle-based performance:}

These efforts monitor the driver's performance in the transportation hardware systems such as, truck lane deviation, or steering or speed variability. All of these systems assume that the driver behaviour or the vehicle behaviour deviates from their nominal behaviours when a driver is in fatigue. The measurements include driver steering wheel movements, systems measuring driver's acceleration system on braking, gear changing, lane deviation, and distances between vehicles [2].

\subsection{In-vehicle on-line driver's status monitoring:}

This category of efforts records online bio-behavioural dimension(s) of an operator, such as parameters characterizing eye movements, head movements, facial expressions, heart activities [22], brain electrical activity, reaction time, electroencephalograph measures (such as EEG) for monitoring brain activity and ocular measures to characterize eyelid movement (such as PERCLOS) and characterize pupil movement (such as saccade movement versus fixation time). Other visual measures include parameters characterizing facial muscles, body postures, and head nodding [2][25].

Different possible sensor positions are shown in figure 1.

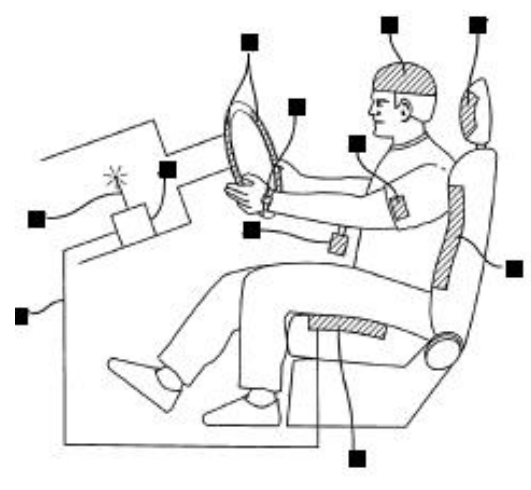

Figure 1. Sensor positions indicated by black squares. [25]

\section{COMPARING DIFFERENT VIGILANCE MODELS}

As discussed in section 2, different vigilance measuring models use different sensor inputs. These models can be compared based on various aspects like intrusiveness, ability to detect sleep earlier, context dependence, cost, scalability, susceptibility to failures etc.

EEG based models[10-20][25] are costly and intrusive as the subject has to put on different electrodes on her head to sense the brain activities but are comparatively more accurate to detect fatigued state earlier as it record brain activities directly. The models based on human-machine interface measures [23] are context dependent as the human-machine interface changes with application. The models based on behavioural measures and visual measures are low cost, nonintrusive but are susceptible to failures as tracking of body movements, eyes, changes in eye parameters is complicated. For example tracking eyes if subject has put on spectacles often fails. Because of required external sensors, most of the models except those based on visual measures are hardly scalable (not applicable for monitoring group vigilance). 


\section{POSSIBLE FUTURE RESEARCH DIRECTIONS}

In this section we discuss future scope for research in the field of vigilance monitoring.

\subsection{Detecting different (non-fatigue) mental states leading to non-vigilance}

Fatigue is not the only reason for non-vigilance. Identifying other possible symptoms of nonvigilance and detecting the same can be possible future work in the said field. For example in vehicle based system, if driver is observed talking continuously, then this may lead to lack of concentration and hence non-vigilance.

\subsection{Vigilance monitoring for mass audience}

Extension to the individual vigilance measuring models to measure the vigilance level of mass audience can be a challenging future work. Training data can be collected through experimentation where visual measures of audience can be sensed and using certain machine learning techniques, co-relation between these measures and actual feedback given by audience can be established. The generated model can be used to find general interesting-ness measure of certain lecture. Some interesting observation about mass audience can be helpful, like vigilance level of certain person also depends on vigilance level of neighbours and this can be modelled as Dynamic Bayesian Network similar to work in [2].

\subsection{Vigilance preserving techniques}

The effects of different vigilance preserving techniques (like periodic beeps etc) can also be studied experimentally.

\subsection{Tracking face and eyes}

Many attempts for vigilance monitoring are based on sensing eyes and face status. Those methods can be further improved for sensing eyes with varying light conditions or when person is wearing spectacles [1]. Tracking face and eyes will enhance robustness and error tolerance of the system [3].

\section{Conclusions}

This paper reviews research work in the field of vigilance monitoring and also compares fatigue models on different aspects. Some possible future research directions are also discussed in the paper. Modelling vigilance of mass audience is not simply collective vigilance of independent individuals as behaviour of an individual can not be taken as sign of vigilance or non-vigilance unless it is compared with that of others in a group. Therefore new parameters have to be discovered for sensing mass vigilance. As some non-fatigued mental states are also responsible for non-vigilance of an individual, identifying these states and finding required sensors would be a key future research in this field.

\section{REFERENCES}

[1] Bergasa, L.M. Nuevo, J.Sotelo, M.A.Barea, R.Lopez, M.E "Real-time system for monitoring driver vigilance", Intelligent Transportation Systems, IEEE Transactions on March 2006,Volume: 7 Issue:1, On page(s): 63 - 77,ISSN: 1524-9050 
[2] Qiang Ji,Peilin Lan, Carl Looney "A Probabilistic Framework for Modeling and Real-Time Monitoring Human Fatigue”, IEEE Transactions on Systems, Man, and Cybernetics—part A: Systems and Humans, vol. 36, no. 5, september 2006

[3] Guang-Yuan Zhang, Bo Cheng, Rui-Jia Feng, Jia-Wen Li "Real-time driver eye detection method using Support Vector Machine with Hu invariant moments", International Conference on Machine Learning and Cybernetics, Volume: 5, Publication Year: 2008 , Page(s): 2999 - 3004

[4] Mohamad Hoseyn Sigari , "Driver Hypo-Vigilance Detection based on Eyelid Behavior ”, Seventh International Conference on Advances in Pattern Recognition. Year 2009.

[5] Lifang Deng; Xingliang Xiong; Jin Zhou; Ping Gan; Shixiong Deng; "Fatigue Detection Based on Infrared Video Pupillography", 4th International Conference on Bioinformatics and Biomedical Engineering (iCBBE), Publication Year: 2010 , Page(s): 1 - 4

[6] Fabian Friedrichs and Bin Yang, "Camera-based Drowsiness Reference for Driver State Classification under Real Driving Conditions", 2010 IEEE Intelligent Vehicles Symposium, June 21-24, 2010

[7] Hariri, B.; Abtahi, S.; Shirmohammadi, S.; Martel, L.; "Demo: Vision based smart in-car camera system for driver yawning detection" 2011 Fifth ACM/IEEE International Conference on Distributed Smart Cameras (ICDSC), Digital Object Identifier: 10.1109/ICDSC.2011.6042952 Publication Year: 2011, Page(s): 1 - 2

[8] M. Wang, H. P. Chou, C. F. Hsu, S. W. Chen, and C. S. Fuh, "Extracting Driver's Facial Features During Driving ", 2011 14th International IEEE Conference on Intelligent Transportation Systems Washington, DC, USA. October 5-7, 2011

[9] T. D'Orazio, M. Leo, A. Distante, "Eye detection in face images vigilance system", IEEE Intelligent Vehicles Symposium University of Parma Parma, Italy June 14-17,2004

[10] $\mathrm{Mu} \mathrm{Li}$, Jia-Wei Fu and Bao-Liang Lu, "Estimating Vigilance in Driving Simulation using Probabilistic PCA", 30th Annual International IEEE EMBS Conference Vancouver, British Columbia, Canada, August 20-24, 2008

[11] Alessandro Giusti, Chiara Zocchi, and Alberto Rovetta,"A Noninvasive System for Evaluating Driver Vigilance Level Examining Both Physiologicala and Mechanical Data”, IEEE TRANSACTIONS ON INTELLIGENT TRANSPORTATION SYSTEMS, VOL. 10, NO. 1, MARCH 2009.

[12] Li-Chen Shi, Bao-Liang Lu, "Dynamic Clustering for Vigilance Analysis Based on EEG", Proceedings of International Conference of the IEEE Engineering in Medicine and Biology Society, pp. 54-57, Vancouver, BC Canada, Aug. 2008.

[13] Chin-Teng Lin; Yu-Chieh Chen; Ruei-Cheng Wu; Sheng-Fu Liang; Teng-Yi Huang; "Assessment of driver's driving performance and alertness using EEG-based fuzzy neural networks ", IEEE International Symposium on Circuits and Systems, 2005. ISCAS 2005, Issue Date: 23-26 May 2005 On page(s): 152 - 155 Vol. 1, Print ISBN: 0-7803-8834-8

[14] Kai-Quan Shen, Chong-Jin Ong, Xiao-Ping Li, Zheng Hui, and Einar P. V. Wilder-Smith, "A Feature Selection Method for Multilevel Mental Fatigue EEG Classification", IEEE TRANSACTIONS ON BIOMEDICAL ENGINEERING, VOL. 54, NO. 7, JULY 2007

[15] L.M. King, H.T. Nguyen, S. K. L. Lal, "Early Driver Fatigue Detection from Electroencephalography Signals using Artificial Neural Networks", Proceedings of the 28th IEEE EMBS Annual International Conference New York City, USA, Aug 30-Sept 3, 2006

[16] Tian Ouyang, Hong-Tao Lu, "Vigilance Analysis Based on Continuous Wavelet Transform of EEG Signals", 2010 International Conference on Biomedical Engineering and Computer Science (ICBECS), Digital Object Identifier: 10.1109/ICBECS.2010.5462289, Year: 2010 , Page(s): $1-4$

[17] Fu-Chang Lin, Li-Wei Ko, Shi-An Chen, Ching-Fu Chen, Chin-Teng Lin, "EEG-based cognitive state monitoring and predition by using the self-constructing neural fuzzy system", , Proceedings of 2010 IEEE International Symposium on Circuits and Systems (ISCAS), Digital Object Identifier: 10.1109/ISCAS.2010.5536955, Publication Year: 2010, Page(s): 2287 - 2290

[18] Xin-An Fan; Lu-Zheng Bi; Zhi-Long Chen; "Using EEG to detect drivers' emotion with Bayesian Networks", 2010 International Conference on Machine Learning and Cybernetics (ICMLC), Volume: 3, Digital Object Identifier: 10.1109/ICMLC.2010.5580919, Publication Year: 2010 , Page(s): 1177 1181

[19] S. Hanke, J. Zeitlhofer, G. Wiest, W. Mayr and D. C. Moser , "Automated Vigilance Classification based on EOG signals: Preliminary Results", IFMBE Proceedings, 2009, Volume 25/9, 428-431, DOI: $10.1007 / 978-3-642-03889-1 \_115$

[20] David Burton, "Vigilance monitoring system”, U.S Patent No US 6575902 B1, Date of patent 10 June 2003. 
[21] Li Shiwu, Wang Linhong, Yang Zhifa, Ji Bingkui, Qiao Feiyan, Yang Zhongkai, "An Active Driver Fatigue Identification Technique Using Multiple Physiological Features" ,2011 International Conference on Mechatronic Science Electric Engineering and Computer August 19-22, 2011, Jilin, China.

[22] Ikeda Hiroshi, Mihoshi Akihiro, Ikeda Hajimu, Nakaseko Masaru, "Effects of nighttime nap and daytime sleep on heart rate and drowsiness in long-distance highway bus drivers", IATSS Res (Int assoc traffic saf sci),ISSN:0386-1112, vol.30;no.2;page.78-82(2006)

[23] A.V. Desai, M.A. Haque, "Vigilance monitoring for operator safety: A simulation study on highway driving", Journal of Safety Research 37 (2006) 139 - 147

[24] Qiang Ji, Xiaojie Yang, "Real-time eye, gaze, and face pose tracking for monitoring driver vigilance", Journal of Real-Time Imaging, Volume 8 Issue 5, October 2002, ISSN: 10772014, DOI: 10.1006/rtim.2002.0279

[25] Patrick K. Sullivan et al, "Systems and methods for intelligent medical vigilance with alert cause indication", U.S. Patent Publication number: US 2010/0052917 A1, Pub Date 4 march 2010.

[26] Jokinen,Tapio, Saarenpää-Heikkilä, Outi, Laippala, Pekka, "A new visual performance test offers new variables for assessing daytime vigilance: Short pauses in performance", Behavior Research Methods, Springer New York, Volume: 31 Issue: 1 1999-03-01

\section{Authors}

Dr. B. F. Momin completed his B.E. and M.E. in Computer Science and Engg. from Shivaji University, Kolhapur, India in the year 1990 and 2001 respectively. He completed his Ph.D in Computer Science and Engg from Jadavpur University, Kolkata, India. He is currently working as H.O.D. of Computer Engg Department in Walchand College of Engineering, Sangli, India

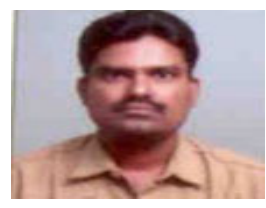

Mr. Parag P. Abhyankar completed his B.Tech (Computer Engg) from Dr. B. A. T. University, Lonere, India in 2002 and M.Tech (CSE) from I.I.T. Bombay in 2010. He is currently working towards his Ph.D under guidance of Dr. B. F. Momin and also working as Assist Professor in department of Information Technology, F.A.M.T. Ratnagiri, India

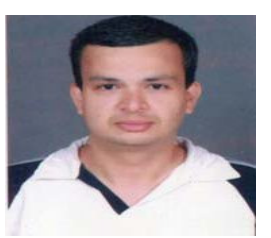

